\title{
A Synopsis Of 3D Printing And Robotics Applications In Construction
}

\author{
Souhail Elhouar, PhD, $\mathrm{PE}^{\mathrm{a}^{*}}, \mathrm{M}$. Ammar Alzarrad, $\mathrm{PhD}^{\mathrm{a}}$, Samar Elhouar ${ }^{\mathrm{b}}$ \\ ${ }^{a}$ Bradley University, Peoria, IL 61625, USA \\ ${ }^{b}$ Graduate Student, Illinois Institute of Technology, Chicago, IL, USA
}

\begin{abstract}
One of the difficulties hindering the application of 3D printing technology in construction is related to the versatility of materials and components used to produce a building or other structure. The prospect of using this technology is further complicated by the sheer size of the edifices to be constructed. While 3D printing a mechanical component can now be done in someone's basement with affordable and readily available equipment, applying the same technology to produce large structures and building components is a challenge. In recent years, researchers have been working towards overcoming this challenge by trying to develop new construction materials and methods that would be more suitable for the application of 3D printing technology. One of the approaches that can be considered is the combination of robotics technology with $3 \mathrm{D}$ printing to automate construction activities. The use of robots in construction has been proposed long before 3D printing became possible or known but never gained widespread construction site usage, mainly because of the difficulty associated with the automation of most construction tasks. However, the combination of $3 \mathrm{D}$ printing with robotics may be the way to change that. In this paper, the authors examine the suitability of $3 \mathrm{D}$ printing in a number of construction tasks and present ideas that modify established construction methods to make them more suitable for automation. The authors then examine how the introduction of robotics in conjunction with 3D printing to the construction site may make it possible to automate a number of construction tasks. Some of the benefits of such automation include lower safety risks, improved control over construction schedules, more economical construction, and a better ability to build in remote areas and challenging environments.
\end{abstract}

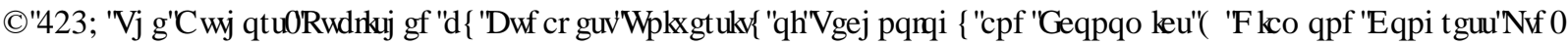

Peer-review under responsibility of the scientific committee of the Creative Construction Conference 2019.

Keywords: 3 d printing, construction automation, construction technology, robotics;

\section{Introduction}

Back in 1986, Whittaker [1] described construction robotics as an "infant discipline whose mature form is not apparent." More than thirty years later, this statement still holds true in spite of significant advancements in computer and automation technologies. Although robots have been quite successfully used for decades in areas such as manufacturing, commerce, space, and marine exploration, and other fields, their use in construction has not been as widespread as many in the industry would have wanted it to be. This is by no means due to the lack of foresight or effort on the part of researchers and professionals in the industry. In fact, the idea of incorporating automation in the construction process has been floating around for a long time and may have started even before researchers such as the 
Elhouar, Alzarrad, \& Elhouar/ Proceedings of the Creative Construction Conference (2019) 105 https://doi.org/10.3311/CCC2019-105

ones referenced in this paper started reporting on it. Garbotz [2] investigated the needs in capital for shifting manual work to machines in order to increase productivity and was among the first researchers to address the issue of automation in the construction industry. Klein and Patterson [3] studied the possible use of multiple-armed robots in the construction of large space structures, and they described a successful effort to simulate the locomotion of a vehicle with arms that was modeled after currently (1982) available industrial manipulators. Hasegawa [4] reported that the first appearance of building construction robots in Japan took place in the mid-1970's and mentioned six pioneering research projects that were carried out between 1978 and 1979. These projects investigated the automation of activities such as wooden panel wall assembly, tall building surface panel assembly, and other operations. Hasegawa [4] reiterated the need for developing new robot engineering technology for the construction industry and went on to propose a conceptual design of a robotized construction system, which represented the basic idea of today's 3D printing technology. Hasegawa [4] concluded that, in order to accomplish the desired automation, a large task force with sufficient financial support and time will be needed to successfully study all the required development topics.

In a book that was published in 1988, Yates [5] predicted that, by the turn of the twentieth century, there was good potential for increased use of self-directed robots controlled by expert systems. At the time, some automation and robotics technology was already being used to finish concrete, spray-paint buildings, or carry out certain tasks in hazardous environments. Drazan [6], one of the early researchers who investigated the use of robots in the construction industry, divided the potential robotics application areas in construction into three groups, which include the automation of existing equipment, the automation of existing processes, and the use of robots for existing manual processes. These proposed approaches might have worked for some aspects of the construction industry including tunneling and excavation, prefabricated and modular construction, and, more recently, moderately applicable 3D printing. However, they nonetheless proved to be insufficient for bringing significant automation to this considerable sector of the global economy. Balaguer [7] argued that the main difficulty with robotics and automation in construction (RAC) is related to the unstructured nature of construction sites and a number of other issues, which will be discussed in the next section. Balaguer [7] went on to state that there are two types of robotics interventions that can be applied to RAC: hard robotics and soft robotics. In hard robotics, robots are designed to directly automate construction processes. In contrast, soft robotics research aims at integrating software with on-site sensory data acquisition and processing, human operator's safety and security, and chip-based process control among other robotic and automation hardware. Louis et al. [8] weighed in on the reasons behind the contrast between the levels of automation in the various manufacturing industries compared to that in the construction industry. They attributed the disparity to the distinctive nature of construction activities, which are characterized by "customized projects, uncontrolled outdoor work sites, and necessary collaboration between disparate entities." They also noted how the lack of progress of real automation in the industry led to a decline in construction robotics research activities. They cited the findings of Son et al. [9] who studied global trends in research and development of automation and robotics in construction and reported that the percentage of papers categorized under "Construction Robotics" declined from 71\% in 1990 to 33\% in 2008. Louis et al. [8] came to the conclusion that the issue of automation of construction needs a fresh examination in order to fulfil the vision of a fully automated construction worksite. They went on to describe a new approach that enables the automation of entire construction operations by using Discrete Event Simulation (DES) models to orchestrate and actuate robots on the construction site.

Based on this discussion, it is clear that the solution to the construction automation problem needs to go well beyond the automation of existing processes. The construction industry has a lot to gain from integrating more automation in the construction process. Such automation will generate a number of improvements, including faster and more efficient construction methods and processes with better results in terms of safety, quality, and cost. In this paper, the authors first consider some of the challenges that the construction industry has been facing in its effort to incorporate more automation. They then propose an eight-step process in which robotics and $3 \mathrm{D}$ printing in combination with innovative materials and methods of construction can be used to achieve a sustained automation effort. 
Elhouar, Alzarrad, \& Elhouar/ Proceedings of the Creative Construction Conference (2019) 105 https://doi.org/10.3311/CCC2019-105

\section{Drivers for and Challenges to Construction Automation}

It is well established that the construction industry, with a few exceptions, is generally on the slower side when it comes to adopting automation technology. Exceptions to this general observation include the prefabricated and modular construction and the steel fabrication sectors, which have significantly benefited from advances in automation and robotic technologies. In other words, automation primarily benefited all construction related activities that can take place in a controlled, manufacturing-like environment. For most remaining sectors of the industry, construction means and methods have seen little change over time. This is not to say that the industry can afford to continue along this path. Banik and Barnes [10] identified a number of challenges that the construction industry is facing, with the first issue having to deal with an anticipated shortage in resources, including skilled workers and technical and supervisory staff. This would be a major problem that automation can readily solve. Owen et al. [11] suggested that the continued practice of using paper specifications and drawings to define the technical requirements and configurations for construction products does not facilitate the integration of physical work processes. As a result, they maintain that opportunities for making use of appropriate levels of automation are largely lost. This is because this traditional method of design information production and delivery does not promote during the design process the consideration of detailed fabrication and assembly or the best venue for performing certain construction tasks.

Feng et al. [12] identified the rugged, dynamic, and unstructured environment of the construction worksite as well as the uncertainty and evolving sequence of on-site events as unique challenges that stand in the way of widespread use of robotics and soft automation in construction. They noted that the challenge for the robotics industry that wants to cater to the construction sector is to design and build robots that are capable of sensing and adapting to environmental changes, in addition to the ability to replicate basic human motion. Elattar [13] who studied opportunities for automation and robotics in construction stated that one of the challenges that the construction industry will face in the next few decades will be to meet the demand that will result from enormous migration to cities. He cited forecasts indicating the ratio of urban population in the world will go from $45 \%$ in 1995 to $55 \%$ in 2015 . According to data obtained from the World Bank Web Site [14], Elattar's [13] prediction was quite accurate as urban populations around the world increased from about $45 \%$ in 1995 to about 54\% in 2015 and reaching nearly 55\% in 2017. Elattar [13] argued that automation will play a big role in helping the industry meet the increased demand and identified many areas where automation has been tried.

According to Gagliordi [15], Autodesk also weighed in on the population issue by focusing on its global rise, which will lead to increased demand for buildings. This demand, combined with the short supply of qualified workers create a need to change the traditional construction techniques. Autodesk's solution to this problem is a sort of a portable additive manufacturing "toolbox" for the construction industry. The idea is to package various robots and printing systems capable of printing large components in shipping containers and to send them from one job site to another as needed. According to Gagliordi [15], this method could be a solution to the construction labor shortage problem and would enable parts to be produced faster and more accurately. To summarize, it is evident that the idea of fully automating construction processes faces many challenges that the industry has so far not been able to resolve. The uniqueness of each project combined with the variability and rugged nature of construction sites make it unwelcoming to robots, which are best suited to operate in predictable and organized environments. The variability in construction components and their materials, different components that often may need to be installed at the same location within the same timeframe, adds another dimension to the already challenging task of developing viable automation processes. The variation in construction methods between trades and the possibility of them having to operate concurrently creates the need for having multiple robots operating within close proximity from each other. Having machines around the construction site that may make sudden unpredictable moves creates a safety hazard. Also, weather and other environmental factors can have an adverse effect on the performance of robots. And finally the use of robots may present some unique liability issues that need to be investigated and dealt with. For example, when a robot's malfunction causes a project delay or an accident, it may not be very clear if the responsibility should be assumed by contractors, designers, the owner, or the robot's manufacturer or programmer. One can only imagine the ramifications of a situation where a robot causes an accident because it made a wrong decision when its visual analysis system 
Elhouar, Alzarrad, \& Elhouar/ Proceedings of the Creative Construction Conference (2019) 105 https://doi.org/10.3311/CCC2019-105

became impaired by a sudden poor weather condition. However, it may be possible to avoid weather related issues in some projects. For example, Kim et al. [16] proposed a conceptual model for what they called a construction factory (CF) which is a temporary structure that will shield robots from weather and environmental elements during construction.

\section{Automation and Robotics}

Robots can be defined as high performing computer systems that are motion capable and that can be programmed to perform tasks that normally can only be performed by humans. When the tasks to be performed are simple, the robot can consist of a number of mechanical parts that are driven by a simple sequential computer code. This includes, for example, simple repetitive actions such as moving certain objects from one specific location to another, or continuously depositing welding material between prepositioned parts. However, robots with specialized capabilities and Artificial Intelligence (AI) driven software are typically needed to carry out tasks that go beyond simple predefined motion. To put this into perspective imagine that a robot is to assist with the construction of a reinforced masonry wall. The robot's task is to alternate between positioning a course of Concrete Masonry Units (CMU) in place and then following up with the positioning of reinforcing steel. One of the challenges here for the robot is to recognize what it is picking up so it applies the appropriate amount of force to the object without crushing it (too much force) or dropping it (too little force). Moreover, to be able to pick up components and place them where they belong, at a minimum, a robot needs to have the ability to 1) know the item's original location, 2) have a mechanism to safely pick up the item, 3) know the planned item's position, 4) have a mechanism to safely manipulate the item into its final position, and 5) have the ability to adequately attach the item while holding it into place. These minimum requirements will allow the robot to install the item but do not address other important requirements, such as safety for example: robots will also need to have the ability to identify hazards and avoid them. The ability to identify an object and know how to deal with it must rely on sensory attributes that come naturally to people but that robots must be designed to have. To be effective in the construction site, robots need to be aware of their surroundings at every moment and need to be able to recognize objects by relying on technology such as image recognition, radar, laser, and GPS. Luckily for the construction industry, these technologies have been well developed for the automotive and other industries in recent years.

Ardiny et al. [17] conducted a comprehensive review of state-of-the-art research in automated construction by autonomous mobile robots. They identified three general categories of construction robots: tele-operated systems, programmable construction machines, and intelligent systems. Tele-operated systems are machines that are remotely controlled by human operators who interpret data and cognitive activities. Programmable construction machines can be pre-programmed to perform a number of functions that a human operator can access through a menu and direct the machine to execute them. The intelligent systems category encompasses machines that can accomplish tasks in fully autonomous mode (with no human intervention) or semi-autonomous mode (with some level of human interaction). Ardiny et al. [17] concluded that there is a need for more rigorous research related to hazard detection, accurate positioning, human-machine and machine-machine interaction and collaboration, and the development of new materials, components and processes that are more suitable for automation.

\section{Robotics Automation and 3D Printing}

The solution that will lead to meaningful automation in the construction industry must go beyond 3D printing and automating typical construction methods. This is because construction means and methods that were developed around peoples' abilities are not necessarily the best suited for automation. For any effort to be successful in effecting more automation in the construction industry, it must first include an evaluation of the adaptability of prevalent practices to automation, and then it must look into developing new construction methods and materials be able to take full advantage of all the automation technologies that may be available today or in the future. The illustration in Fig. 1 shows a simplistic classification of construction tasks based on their adaptability to automation. Prefabricated components are constructed in volume in a controlled manufacturing environment, typically using a repetitive process. This makes them a prime candidate for automation. 
Elhouar, Alzarrad, \& Elhouar/ Proceedings of the Creative Construction Conference (2019) 105 https://doi.org/10.3311/CCC2019-105

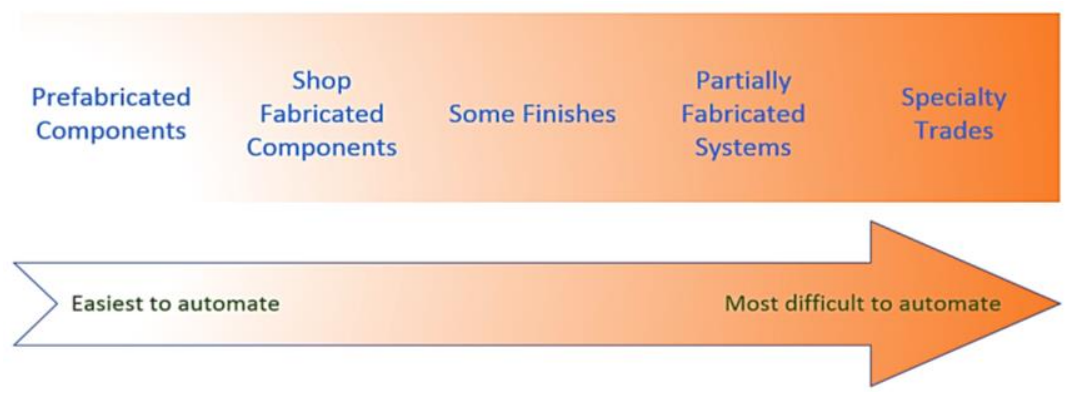

Fig.1. Classification of Construction Tasks Based on their Adaptability to Automation

Next on the list are shop fabricated components. These may include structural steel members and trusses, wood trusses, and HVAC and other similar components that are specifically designed for a project and that can be fabricated in a controlled shop environment by stationary robots. In general, automation has been quite successful in these two categories of construction. The third category in order of increasing difficulty of automation includes the placement of finishes such as painting and placing fireproofing on exposed steel members. These types of activities have the advantage of being well defined in space, but their automation requires the use of robots that are capable of motion. This adds a safety concern and a new dimension to the automation effort as robots that are intended to be able to move must also have the ability to be aware of their surroundings in order for them to avoid hazards.

Partially fabricated systems are those that consist of assemblies of prefabricated or shop fabricated components. For example, steel frames that are made up of assemblies of shop fabricated beams and columns belong to this category. The challenge, in this case, is how to automate the process of lifting the prefabricated or shop fabricated components and attaching them in place. The specialty trades category encompasses all construction activities that deal with electrical, plumbing, mechanical and similar systems, and involving activities that often must be accomplished in confined spaces with many interferences and using special equipment. The ability to achieve satisfactory automation of these activities will require a considerable research and development effort to formulate novel means and methods that are more amenable to automation. The key to achieving a complete and successful automation in construction is to invest in significant research leading to the development of new technologies and construction materials and methods that will help the automation effort to move forward. The construction site of the future could very much look like one where 3D printers and different types of robots work in synch to complete a construction project.

3D printing is a different name for what has been known in the manufacturing sector as additive manufacturing. The process works by having a computer controlled system superimpose layers of material to form a shape for which the geometry is defined by a three-dimensional computer model. There are clear limitations to this process as, for example, it cannot span between parts of the object being printed because there is no material to add to. When the object to be printed is small, a filler material can be used to act as temporary support for the spanning portion of the object and then removed when the object becomes able to support itself. However, this approach will not work well with large building structures. The alternative becomes to prefabricate spanning elements and use robots to install them or to provide temporary support by some other means so the 3D printing can be performed. Specialized robots may also be used to perform tasks such as welding, bolting, painting, or even a combination of tasks. Moreover, new construction methods and processes will need to be developed. For example, large-scale multi-arm robots can be used for the erection of structural steel. This will require some revisions in the methods of fabrication and preparation for erection of structural steel components. An adequately designed robot should be able to pick up a component in its proper sequence of installation and hold it perfectly in place with one arm while other arms weld it or bolt it to its supporting member. This will allow for structural steel members to be erected faster and with more accuracy than if traditional methods are used. For this to work perfectly, changes will need to be introduced to the fabrication process as well to produce structural steel components that can communicate to the robot information about what they are and their sequence of installation. Evidently, the information needs to be finalized during the design process so it can be forwarded to the fabricator along with fabrication details. This reinforces the idea that the success of the automation effort in the 
Elhouar, Alzarrad, \& Elhouar/ Proceedings of the Creative Construction Conference (2019) 105 https://doi.org/10.3311/CCC2019-105

construction industry will require more than just trying to automate current construction practices. The automation process needs to start during the design phase with sophisticated Building Information Modeling (BIM) models that capture each little detail of the project. There are many areas where construction means and methods need to be reinvented for successful automation and one more example that the authors wish to present in this paper has to do with the construction of reinforced concrete members. If one is to consider the construction of a reinforced concrete beam, the traditional method is to assemble the reinforcing steel bars and stirrups in the shop or on site, construct the formwork, and place the reinforcing steel in the forms in such a way that it lines up adequately with the reinforcement of other connecting members. Once workers verify that the reinforcement is placed in such a way that concrete cover requirements are met, the concrete pouring process can be initiated to complete the beam. This intricate process is clearly not suitable for automation. However, with some modifications that take into consideration the accuracy that can be achieved from concrete 3D printing and the advanced capabilities of current robots, it becomes possible to automate the construction of a reinforced concrete beam by following a different process.

In this regard, the authors wish to introduce an eight-step process for the automatic construction of reinforced concrete members. The proposed process assumes that the supporting columns have already been 3D-printed leaving room for continuing reinforcement to be doweled in. Since the 3D-printing process is achieved by adding layers of material that hold their shape, only a platform will need to be constructed to support the beam during construction. This will save the effort of having to build a complete set of formwork for the beam. Once the platform is in place, the portion of concrete just below the main bottom reinforcing steel is 3D-printed, leaving an empty space for the rebar that will eventually be inserted into the column. At this point, a picking and placing robot places the preassembled bottom reinforcing steel in place. In this step, the bars are connected together at only a number of points to make it easy for the robot to pick them up and position them accurately. Next, the part of the beam between the bottom and top reinforcements is 3D printed. When additional longitudinal reinforcement is required anywhere in the beam, it is simply placed by the picking and placing robot when the 3D-printing reaches the appropriate elevation. The 3Dprinting process in this case leaves open areas in the beam to allow for shear reinforcement, top vertical reinforcement, and column rebar or continuation dowels to be inserted. The next phase involves the placement of the top reinforcing bars and the shear reinforcement stirrups by the picking and placing robot. In this step, the shear reinforcement stirrups are inverted U-shaped pieces of steel that a robot can easily drop into the space that was left empty, in order, and using the design specified spacing. Following this, the 3D-printing robot uses a special bonding compound to fill the space that was initially left empty and now is partially occupied with stirrups. The nature and composition of the bonding compound is something that will need to be investigated by the concrete materials research community. The final stage of the beam construction process would be for the portion of concrete above the top reinforcement to be 3D-printed.

\section{Conclusions}

The world is facing many infrastructure challenges that need to be readily addressed and the construction industry must position itself to meet those challenges. Faced with a shrinking labor force and the requirement to execute more complicated projects with increasingly limited resources, the industry has to intensify its effort towards significantly incorporating more automation into its processes. The computer information and robotic technologies have come a long way in the last few years and there are many advanced technological tools available today that can be used by the construction industry to improve and automate its processes in all of its sectors. However, significant construction automation research needs to be done to achieve this objective because the difficulties that are associated with this endeavor are very unique and specific to construction, and many of the automation techniques that have been working well for other industries are just not suitable for automating most construction activities. In this paper, the authors made the case for the need of a rigorous research effort in construction materials and construction means and methods to be able to take full advantage of robotics and 3D-printing technologies. It must also be pointed out that a successful automation of construction activities will not be achieved if construction methods are not considered during the design process. Building information models must be expanded to include detailed construction related information, and a platform for integrating Building Information Modeling (BIM) with Construction Information Modeling (CIM) must be created. 
Elhouar, Alzarrad, \& Elhouar/ Proceedings of the Creative Construction Conference (2019) 105

https://doi.org/10.3311/CCC2019-105

\section{Acknowledgements}

The research leading to the publication of this article was partially supported by the Department of Civil Engineering and Construction at Bradley University in Peoria, Illinois, United States of America.

\section{References}

[1] Whittaker, W. L. (1986) Construction Robotics: A Perspective. In: CAD and Robotics in Architecture and Construction, pp 105-112, Springer, Boston, MA

[2] Garbotz, G. (1969) Mechanization and Productivity in Building Industry, Foerdern u Heben Volume 19, Issue 2, Feb, Pages 74-81, Elsevier

[3] Klein, Charles A. and Patterson, Mark R. (1982) Computer Coordination Of Limb Motion For Locomotion Of A Multiple-Armed Robot For Space Assembly, IEEE Transactions on Systems, Man and Cybernetics, Volume SMC-12, Issue 6, Nov-Dec, pp 913-919, https://doi.org/10.1109/TSMC.1982.4308927

[4] Hasegawa Y. (1986) Robotization of Reinforced Concrete Building Construction in Japan. In: CAD and Robotics in Architecture and Construction, pp 113-121, Springer, Boston, MA, https://doi.org/10.22260/ISARC1986/0032

[5] Yates, A. P. (1988) Technological Advances in the Construction Sector. In Globalization of Technology: International Perspectives, pp 68-79, The National Academics of Sciences, Engineering, Medicine, NAP, https://www.nap.edu/read/1101/chapter/9

[6] Drazan P.J. (1986) Application of Robots in Construction Industry: Navigation of a Mobile Robot, Robotic Welding of Steel Bridge Girders. In: CAD and Robotics in Architecture and Construction, pp 225-231, Springer, Boston, MA , https://doi.org/10.1007/978-1-4684-7404-6_22

[7] Balaguer, C. (2004) Nowadays trends in robotics and automation in construction industry: Transition from hard to soft robotics. In 21st International Symposium on Automation and Robotics inConstruction (ISARC). Jeju. Korea. https://doi.org/10.22260/ISARC2004/0009

[8] Louis, J., Dunston, P., and Martinez, J. (2014) Automating Construction Operations using Discrete Event Simulation Models, Construction in a Global Network - Proceedings of the 2014 Construction research Congress, pp. 1043-1052, ASCE.

[9] Son, H., Kim, C., Kim, H., Han, S. H., and Kim, M. K. (2010 Trend analysis of research and development on automation and robotics technology in the construction industry, KSCE Journal of Civil Engineering, 14(2), 131-139 https://doi.org/10.1007/s12205-010-0131-7

[10] Banik, G. C., Barnes, W. C. (2002) Issues and Challenges for the Construction Community, ASEE Annual Conference proceedings, pp. 5365-5372, ASEE.

[11] Owen, R., Amor, R., Palmer, M., Dickinson, J., Tatum, C. B., Kazi, A. S., Prins, M., Kiviniem, A, and Arto, E. B. (2010) Challenges for integrated design and delivery solutions, Architectural Engineering and Design Management, Special Issue: Integrated Design and Delivery Solutions, pp. 232-240, November 01, https://doi.org/10.3763/aedm.2010.IDDS1

[12] Feng, C., Frediricks, N., and Kamat, V.R. (2013) Human-Robot Integration For Pose Estimation and Semi-Autonomous Navigation on Unstructured Construction Sites, ISARC 2013, 30 ${ }^{\text {th }}$ International Symposium on Automation and Robotics in Construction and Mining, Held in Conjunction with the $23^{\text {rd }}$ World Mining Congress, pp. 1317-1325, Montreal, Canada. https://doi.org/10.22260/ISARC2013/0148

[13] Elattar, S. M. S. (2008) Automation and Robotics In Construction: Opportunities And Challenges, Emirates Journal for Engineering Research, 13 (2), pp. 21-26

[14] World Bank Website, https://data.worldbank.org/indicator/SP.URB.TOTL.IN.ZS?end=2015\&start=2008, accessed March $29^{\text {th }}, 2019$.

[15] Gagliordi, Natalie (2018) How Autodesk plans to bring 3D printing robots to construction sites, https://www.zdnet.com/article/howautodesk-plans-to-bring-3d-printing-robots-to-construction-sites/, posted November 8, 2018.

[16] Kim, Dae-Won, An, Sung-Hoon, Cho, Hunhee, Jeong ,Jae-Weon, Lee, Bo-Hyeong, Doh , Nakju Lett, and Kang, Kyung-In (2009) Development of conceptual model of construction factory for automated construction, Building and Environment, Volume 44, pp1634-1642, Elsevier https://doi.org/10.1016/j.buildenv.2008.10.009

[17] Ardiny, H, Witwicki, S., and Mondada, F. (2015) Construction Automation with Autonomous Mobile Robots: A Review, Proceedings of the $3^{\text {rd }}$ RSI International Conference on Robotics and Mechatronics October 7-9, 2015, Tehran, Iran https://doi.org/10.1109/

ICRoM.2015.7367821 